\title{
Monitoring of Functioning Status in Subjects With Chronic Stroke in South Korea Using WHODAS II
}

\author{
Su-Yeon Kwon, MD, Sang-Eun Hong, MD, Ee-Jin Kim, MD, Chang-Hwan Kim, MD, PhD, \\ Kyung-Lim Joa, MD, PhD, Han-Young Jung, MD, PhD \\ Department of Physical and Rehabilitation Medicine, Inha University School of Medicine, Incheon, Korea
}

\begin{abstract}
Objective To follow up the long-term functioning in a community through assessing personal background and status based on the International Classification of Functioning, Disability and Health (ICF) after a stroke, by using a Korean version of World Health Organization Disability Assessment Scale II (K-WHODAS II).

Methods We surveyed 146 patients diagnosed at the first-onset of acute stroke and discharged after Inha University Hospital, and 101 patients answered the K-WHODAS II survey. We analyzed the relationship of six functioning domains of K-WHODAS II with K-MMSE (Korean version of Mini-Mental State Examination) and K-MBI (Korean version of Modified Barthel Index) at admission and discharge, and personal background. All subjects were divided into five groups, according to the disease durations, to assess the functional changes and the differences of K-MMSE and K-MBI at the admission and discharge.

Results K-MBI and K-MMSE at admission and discharge showed no significant differences in all five groups, respectively ( $p>0.05$ ), reflecting no baseline disparity for long-term follow-up. All subjects showed positive gains of K-MBI and K-MMSE at discharge $(\mathrm{p}<0.05)$. The six functioning domains and total scores of K-WHODAS II had decreasing trends until 3 years after the stroke onset, but rose thereafter. Higher scores of K-MBI and K-MMSE, younger age, women, working status, higher educational level, and living with a partner were correlated with lower scores of K-WHODAS II ( $\mathrm{p}<0.05)$.

Conclusion The long-term functioning after stroke was affected not only by cognitive and motor status in hospital, but also by certain kinds of personal background. K-WHODAS II may be used to monitor functioning status in a community and to assess personal backgrounds in subjects with chronic stroke.
\end{abstract}

Keywords Stroke, Functioning, Activity, Participation, Assessment

Received April 16, 2015; Accepted August 3, 2015

Corresponding author: Han-Young Jung

Department of Physical and Rehabilitation Medicine, Inha University School of Medicine, 27 Inhang-ro, Jung-gu, Incheon 22332, Korea Tel: +82-32-890-2486, Fax: +82-32-890-2486, E-mail: rmjung@inha.ac.kr

(c) This is an open-access article distributed under the terms of the Creative Commons Attribution Non-Commercial License (http://creativecommons.org/ licenses/by-nc/4.0) which permits unrestricted noncommercial use, distribution, and reproduction in any medium, provided the original work is properly cited. Copyright $\odot 2016$ by Korean Academy of Rehabilitation Medicine 


\section{INTRODUCTION}

A stroke is a major cause of severe chronic disabilities with a high prevalence in the elderly. The Korean National Health Insurance Corporation reported in 2011 that cardiovascular diseases are the second most common disease in the elderly from 65 to 74 years of age. Also, about a half of stroke patients suffer life-long disabilities in spite of relatively successful acute management and advanced multidisciplinary treatments [1]. Consequently, several Western studies had reported on the functioning status after stroke. Some studies monitored the long-term changes of functioning after stroke [2], and some conducted World Health Organization Disability Assessment Scale II (WHODAS II) surveys to evaluate the functioning status of subjects with chronic stroke in a community $[3,4]$ regarding its conceptual basis in the International Classification of Functioning, Disability and Health (ICF) and a holistic approach to the assessment of impairments and disabilities, as well as activities of daily living (ADL) scales [3].

WHODAS II was recommended by World Health Organization in 2001 for evaluating the ADL of people with chronic disabilities $[5,6]$. WHODAS supports and complements the concept of ICF regardless of the types of diseases [3], and its validity and reliability have been proven.

In South Korea, however, there has been no study on the effects of personal background on the long-term functioning of subjects with chronic stroke in a community. Moreover, there has been no study on the subjects with chronic stroke that used WHODAS II. In South Korea, WHODAS has been only used for psychiatric and rheumatological conditions until recently. In addition, there has also been no study on the long-term functioning of subjects with chronic stroke in relation to cognition, independence in daily living, and disease durations.

In this study, we aimed to follow up on the long-term functioning of subjects with chronic stroke by using WHODAS II. We also collected the scores of initial and discharge scores of the Korean version of the Mini Mental Status Examination (K-MMSE), and the Korean version of Modified Barthel Index (K-MBI), and evaluated the effect of cognition and independence on daily living on longterm functioning.

\section{MATERIALS AND METHODS}

\section{Enrollment of subjects}

There were 146 subjects with chronic stroke, who were diagnosed at their first-onset acute stroke at Inha University Hospital from 2010 to 2013, and also discharged after they were initially enrolled in comprehensive rehabilitative programs. In total, 101 patients agreed to participate in the survey with the K-WHODAS II (Korean version of WHODAS II).

We retrospectively reviewed their medical records and gathered data on the scores of K-MMSE and K-MBI at admission and discharge, to evaluate functional outcomes in hospital and to assess their relationship with the functioning after discharge. The time of admission was set as the first day the patients were transferred to department of physical and rehabilitation medicine. The time of discharge was set as the last day when the functioning status was evaluated before the discharge, which was mostly within one week from the discharge date.

\section{Survey with K-WHODAS II}

Furthermore, we surveyed the patients with the KWHODAS II, which consisted of 12 items. We asked 12 questions on the ADL of subjects with chronic stroke in a community [7], and on personal background, such as gender, age, marital status, educational levels, working status, and marital status. We informed the patients or families about the purpose of the study and also conducted the survey at the outpatient clinic or by phone.

The K-WHODAS II consisted of two main criteria. The first part was about personal background including gender, age, marital status, educational level, and working status. The second part was composed of 12 questions about six domains: two questions for each domain of understanding, getting around, self-care, getting along with people, life activity, and participation. Each question had to be answered according to a 5-Likert scale ( 1 'no difficulty' to 5 'cannot do'). The total scores of six functioning domains ranged between 12 and 60, with lower scores indicated better functioning. The severity of each domain was graded according to the existing standard; total scores of 1-4 belong to mild disability, 5-9 moderate disability, and 10-48 severe disability [8]. 


\section{Modifications of the items}

In this study, we modified the sub-items of the category of working status and marital status according to what we aimed to investigate. The original version of WHODAS II divided the working status into pain employment, selfemployed, unpaid employment, students, housewives, retired, unemployed, and others. The marital status originally included never married, married, separated, divorced, widowed, and cohabiting.

In this study, we defined paid job to include employment and self-employed because they were both currently working statuses with remuneration. Non-paying job was defined to include unpaid work, such as students and housewives because they had a current working status but had no remuneration (Table 1).

Table 1. Demographics

\begin{tabular}{lccc}
\hline & Male & Female & Total \\
\hline Gender & 54 & 47 & 101 \\
Age (yr) & $57.17 \pm 15.39$ & $64.83 \pm 12.45$ & $60.73 \pm 14.55$ \\
Types of stroke & & & \\
\hline Ischemic & 29 & 25 & 54 \\
\hline Hemorrhagic & 25 & 22 & 47 \\
Education (yr) & & & \\
\hline None & 1 & 6 & 7 \\
6 & 8 & 21 & 29 \\
\hline 9 & 9 & 4 & 13 \\
\hline 12 & 20 & 13 & 33 \\
\hline 16 & 13 & 6 & 19 \\
Occupation & & & \\
\hline Paid job & 12 & 3 & 15 \\
\hline Non-paying job & 2 & 10 & 12 \\
\hline No job & 24 & 23 & 47 \\
\hline Retired & 12 & 6 & 18 \\
\hline Others & 4 & 5 & 9 \\
\hline Marriage & & & \\
\hline Never married & 2 & 5 & 7 \\
\hline Married & 39 & 30 & 69 \\
\hline Divorced & 7 & 1 & 8 \\
\hline Separated & 4 & 2 & 6 \\
\hline Widowed & 1 & 10 & 11 \\
\hline Cohabiting & 0 & 0 & 0 \\
\hline Values are prent & & & \\
\hline
\end{tabular}

Values are presented as number or mean \pm standard deviation.
We further modified the multiple sub-items into dichotomous variables to find out whether the presence of job and a cohabiting companion affected the functioning status. Having a job included paid job and non-paying job; having no job included the retired and unemployed. Living with a partner included being married; living

Table 2. Comparisons of total scores of K-WHODAS II according to differences in personal background

\begin{tabular}{|l|l|}
\hline & K-WHODAS II \\
\hline Age (yr) & $26.98 \pm 14.06^{\mathrm{a})}$ \\
\hline$<65$ & $38.23 \pm 16.39^{\mathrm{a})}$ \\
\hline$\geq 65$ & \\
\hline Gender & $35.87 \pm 16.56^{\mathrm{b})}$ \\
\hline Male & $28.20 \pm 14.79^{\mathrm{b})}$ \\
\hline Female & \\
\hline Education $(\mathrm{yr})$ & $51.14 \pm 10.38^{\mathrm{c})}$ \\
\hline None & $36.74 \pm 17.87^{\mathrm{c}, \mathrm{d})}$ \\
\hline 6 & $33.09 \pm 14.23^{\mathrm{cdd}}$ \\
\hline 9 & $28.97 \pm 12.54^{\mathrm{cdd}}$ \\
\hline 12 & $18.80 \pm 11.63^{\mathrm{c}, \mathrm{d})}$ \\
\hline 16 & \\
\hline Occupation & $20.00 \pm 10.77^{\mathrm{e})}$ \\
\hline Having a job & $36.07 \pm 15.51^{\mathrm{e})}$ \\
\hline Having no job & \\
\hline Marriage & $29.95 \pm 15.28^{\mathrm{f})}$ \\
\hline Living with a partner & $37.32 \pm 17.24^{\mathrm{f})}$ \\
\hline Living without a partner
\end{tabular}

Values are presented as mean \pm standard deviation; ttest and ANOVA with post-hoc analysis by Duncan and Tukey.

K-WHODAS II, Korean version of World Health Organization Disability Assessment Scale II.

${ }^{a)} \mathrm{p}<0.05$, comparisons of total scores of K-WHODAS II between different years of age.

${ }^{b)} p<0.05$, comparisons of total scores of K-WHODAS II between different genders.

${ }^{c, d)} \mathrm{p}<0.05$, comparisons of total scores of K-WHODAS II among different years of education received.

${ }^{e} \mathrm{p}<0.05$, comparisons of total scores of K-WHODAS II between different working status (having a job included waged and unwaged vocations, housewives, students, and volunteers).

${ }^{\mathrm{f})} \mathrm{p}<0.05$, comparisons of total scores of K-WHODAS II between different marital status (living with a partner included married status, and living without a partner included unmarried, divorced, and widowed statuses). 
without a partner included never married, separated, divorced, and widowed. Then we compared the total scores of K-WHODAS II between two groups (Table 2).

\section{Subgroups of the subjects}

To assess whether the K-MMSE and the K-MBI at the admission and discharge were different according to the disease durations, we further divided subjects with chronic stroke into five subgroups according to the time elapsed after the onset of stroke: shorter than 6 months (group I), 7-12 months (group II), 13-24 months (group III), 25-36 months (group IV), and 37-48 months (group V) (Fig. 1).

\section{Statistics}

Statistical analyses were performed with SPSS ver. 18 for Windows (SPSS Inc., Chicago, IL, USA). The Student t-test was applied to compare the scores of the K-MMSE and the K-MBI at admission and discharge. The Student ttest and ANOVA were applied to compare the total scores of K-WHODAS II between different statuses of personal background. A Spearman correlation coefficient analysis was applied to evaluate the relationships between the KMMSE, K-MBI, and personal background with K-WHODAS II. In addition, the ANOVA was applied to compare the functioning domains among the five groups and to assess the trends of the K-WHODAS II changes among the five groups after stroke onset. The statistical significance was below $0.05(\mathrm{p}<0.05)$.

\section{RESULTS}

\section{Participants}

Initially, 146 stroke patients were enrolled. Among them, 45 patients were excluded because there was a loss of contact with 34 , and an additional 11 deaths before the survey. Consequently, 101 patients answered the KWHODAS II questionnaire. Fifty-four patients had an ischemic stroke and 47 had a hemorrhagic stroke.

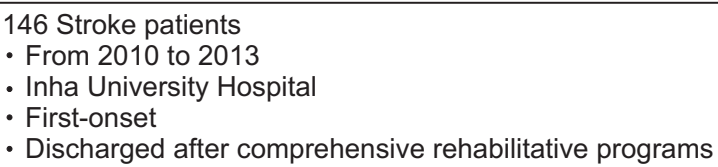

K-WHODAS II

- Personal backgrounds

- Age

- Sex

- Education

- Occupation

- Marriage

- Six functioning domains

- Understanding

- Getting around

- Self-care

- Getting along

with people

- Life activity

- Participation

- Student t-test
Surveyed with K-WHODAS II

- 45 Patients were excluded

- 34 Contact loss

- 11 Deaths before the survey

\section{- Spearman's correlation} coefficient

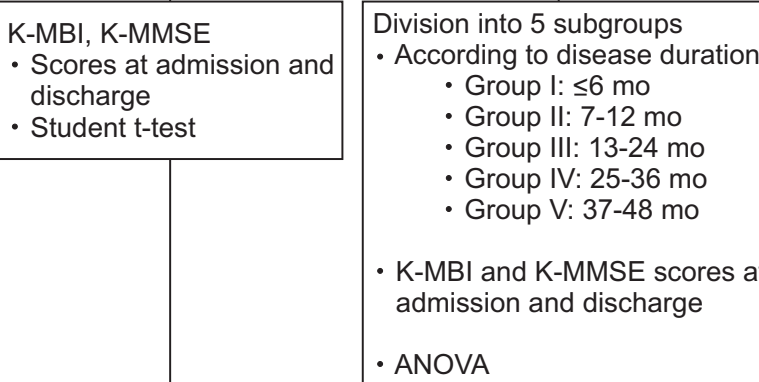

ANOVA
Fig. 1. The flow chart of subject enrollment and statistical analysis. K-WHODAS II, World Health Organization Disability Assessment Scale II; K-MBI, Korean version of Modified Barthel Index; KMMSE, Korean version of MiniMental State Examination. 


\section{Personal background}

Forty-seven patients were female and 54 were male. The mean age of the patients was 60.7 years (women, 64.83 years; men, 57.17 years). Sixty-nine of the patients were married, 8 divorced, 13 unmarried, and 11 widowed. The educational level differed as 29 patients had primary school education; 13, middle school education; 33, high school education, and 19, tertiary education, whereas 7 received no formal education. Sixty-five patients had no jobs at the time of survey, and 27 patients were employed, including unpaid jobs, such as housewives and volunteers (Table 1 ).

The influence of the personal background (e.g., age, gender, educational level, working status, and marital status) on the K-WHODAS II was assessed. The elderly showed higher scores on the K-WHODAS II (26.98 in <65 years and 38.23 in $\geq 65$ years; $p<0.05$ ). Women showed lower scores on the K-WHODAS II (male 35.87 and female 28.20; $\mathrm{p}<0.05)$. Higher educational levels positively influenced the K-WHODAS II (no education 51.14, up to 6 years $36.74,9$ years 33.09, 12 years 28.97 , and more than

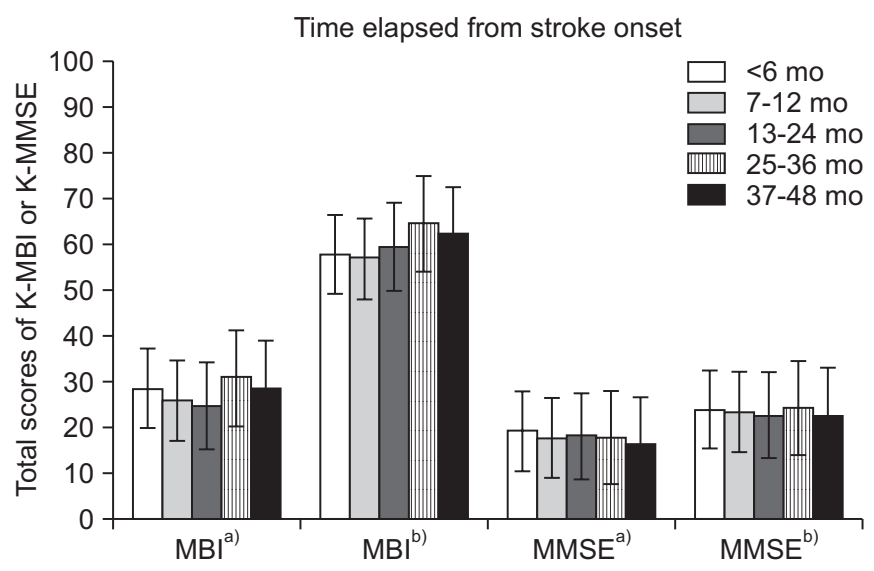

Fig. 2. Comparison of each K-MBI and K-MMSE of five groups at admission and discharge. The discharged patients were divided into five groups according to the time elapsed from the onset of stroke to when they completed the K-WHODAS II questionnaire. These five groups showed no significant difference as we compared their K-MBI and K-MMSE at admission and discharge, respectively ( $p>0.05$ ). ANOVA was applied to compare functioning domains among the five groups. ${ }^{\text {a) }}$ At admission, ${ }^{\text {b) }}$ at discharge. K-MBI, Korean version of Modified Barthel Index; K-MMSE, Korean version of Mini-Mental State Examination; K-WHODAS II, Korean version of World Health Organization Disability Assessment Scale II.
16 years 18.80; $\mathrm{p}<0.05)$. Having a job, including paid and unpaid employment, housewives, students, and volunteers, positively influenced the K-WHODAS II (having a job 20.00 and having no job 36.07 ; $\mathrm{p}<0.05$ ). Living with a partner, which included married status, positively influenced the K-WHODAS II (29.95 in those who lived with partners and 37.32 in those without partners; $p<0.05$ ) (Table 2).

The influence of disease duration on the long-term functioning

We subdivided the discharged patients into five groups according to the time elapsed from the onset of stroke to when they completed the K-WHODAS II survey. Eleven patients were less than 6 months after the onset of stroke, 26 patients between 7-12 months, 33 patients between 13-24 months, 18 patients between 25-36 months, and 13 patients between $37-48$ months. These five groups showed no significant difference as we compared their K-MBI and K-MMSE at admission and discharge, respectively (p>0.05) (Fig. 2).

\section{Functional status in hospital}

We compared the K-MBI and the K-MMSE on admission and discharge, and all stroke patients showed significant improvements on the K-MBI and the K-MMSE at

Table 3. K-MBI and K-MMSE at admission and discharge

\begin{tabular}{|c|c|c|}
\hline & K-MBI & K-MMSE \\
\hline \multicolumn{3}{|l|}{ Ischemic } \\
\hline Admission & $30.23 \pm 23.65^{*}$ & $19.00 \pm 9.66^{*}$ \\
\hline Discharge & $63.29 \pm 26.79 *$ & $22.86 \pm 7.56^{*}$ \\
\hline Gain & $31.97 \pm 18.21$ & $7.42 \pm 6.72$ \\
\hline \multicolumn{3}{|l|}{ Hemorrhagic } \\
\hline Admission & $28.52 \pm 20.42^{*}$ & $18.48 \pm 15.87^{*}$ \\
\hline Discharge & $59.72 \pm 25.83^{*}$ & $23.39 \pm 7.77^{*}$ \\
\hline Gain & $31.30 \pm 19.32$ & $3.68 \pm 4.21$ \\
\hline \multicolumn{3}{|l|}{ Total } \\
\hline Admission & $29.79 \pm 22.18^{*}$ & $18.66 \pm 12.11^{*}$ \\
\hline Discharge & $61.66 \pm 25.00^{*}$ & $23.16 \pm 7.47^{*}$ \\
\hline Gain & $33.82 \pm 19.66$ & $5.89 \pm 6.13$ \\
\hline
\end{tabular}

Values are presented as mean \pm standard deviation.

K-MBI, Korean version of Modified Barthel Index; KMMSE, Korean version of Mini-Mental State Examination.

${ }^{*} \mathrm{p}<0.01$. 
discharge $(\mathrm{p}<0.05)$ (Table 3).

\section{Long-term functioning and contributive factors}

We also assessed the correlations between the total scores of K-WHODAS II with the K-MBI, the K-MMSE, and personal background. The K-MBI and the K-MMSE had correlations with the total scores of K-WHODAS II. The K-MMSE scores at discharge showed the strongest correlation with total scores of K-WHODAS II ( $\mathrm{r}=-0.510)$; then K-MBI at admission ( $\mathrm{r}=-0.480)$, the K-MBI at discharge $(\mathrm{r}=-0.477)$, and the K-MMSE at admission ( $\mathrm{r}=-$ $0.365)$, respectively $(\mathrm{p}<0.05)$. Concerning personal background, the educational level and having a job also had a strong correlation with the total scores of K-WHODAS II ( $r=-0.421$ and $r=-0.484$, respectively; $\mathrm{p}<0.05)$. Furthermore, those younger than 65 years old and women had a correlation with lower scores of K-WHODAS II ( $\mathrm{r}=0.436$ and $\mathrm{r}=-0.225$, respectively; $\mathrm{p}<0.05$ ). Whether or not one lived with a partner did not have a correlation with the total scores of K-WHODAS II ( $\mathrm{r}=0.179, \mathrm{p}>0.05)$ (Table 4).

The changes of long-term functioning status

The trends of the changes of the K-WHODAS II among

Table 4. Correlation of the total scores of K-WHODAS II with personal background, and K-MBI and K-MMSE

\begin{tabular}{lcc}
\hline & \multicolumn{2}{c}{ Total sores of K-WHODAS II } \\
\cline { 2 - 3 } & $\mathbf{r}$ & p-value \\
\hline $\mathrm{K}-M B I^{\mathrm{a})}$ & $-0.477^{*}$ & 0.000 \\
K-MMSE $^{\mathrm{a})}$ & $-0.365^{*}$ & 0.000 \\
K-MBI $^{\mathrm{b}}$ & $-0.479^{*}$ & 0.000 \\
K-MMSE $^{\mathrm{b})}$ & $-0.510^{*}$ & 0.000 \\
\hline Age & $0.436^{*}$ & 0.000 \\
\hline Gender & $-0.225^{*}$ & 0.024 \\
\hline Education & $-0.421^{*}$ & 0.000 \\
\hline Occupation & $-0.484^{*}$ & 0.000 \\
\hline Marital status & 0.179 & 0.074 \\
\hline
\end{tabular}

Values are presented as correlation efficient between the total scores of K-WHODAS II with other variables; Spearman correlation matrix.

K-WHODAS II, Korean version of World Health Organization Disability Assessment Scale II; K-MBI, Korean version of Modified Barthel Index; K-MMSE, Korean version of Mini-Mental State Examination

${ }^{\text {a) }}$ At admission.

${ }^{b)}$ At discharge.

${ }^{*} \mathrm{p}<0.05$. the five groups after stroke onset were analyzed. The sustaining trends of the scores of each of the six functioning domains of the K-WHODAS II and the sum of them (total scores of K-WHODAS II) were maintained until about 3 years after the stroke onset, but declined thereafter. Group I (less than 6 months since stroke onset) showed

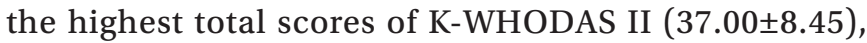
whereas group IV (25-36 months) showed the lowest total scores of K-WHODAS II (27.61 \pm 17.79$)$. Group V (36-48 months) showed increased total scores of K-WHODAS II (38.77 \pm 11.34$)$ compared to group IV. The difference of scores between groups I and IV was about 9.84, and that between groups IV and V was 11.15. However, all these differences had a p-value $>0.05$. Therefore, these score changes, as in functional changes, were not statistically significant but only trends. The social participation, which was one of five domains of the K-WHODAS II, showed an exceptionally earlier decline; it was maintained until 13-24 months but declined from 25 months after onset (Fig. 3).

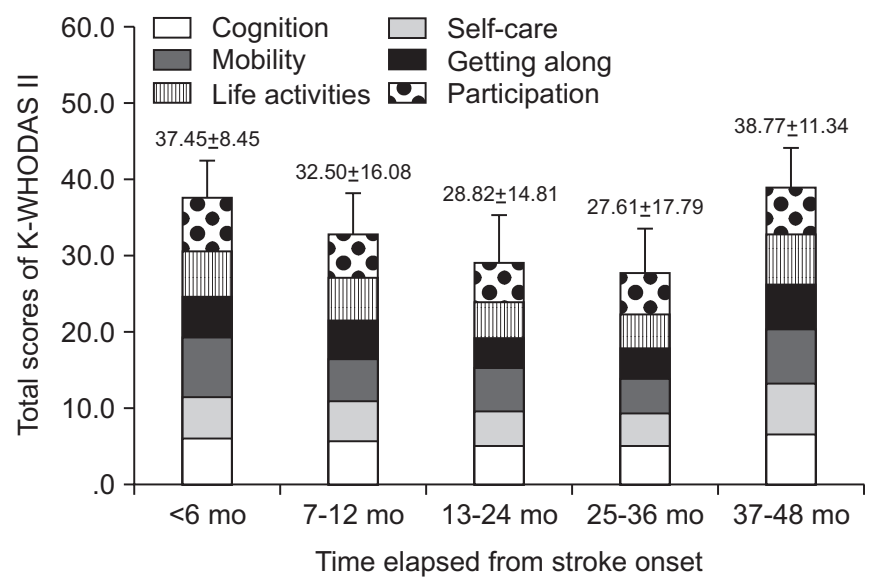

Fig. 3. Long-term follow-up of K-WHODAS II (Korean version of World Health Organization Disability Assessment Scale II) after stroke onset. The trends of the KWHODAS II changes among the five groups after stroke onset were analyzed. The sustaining trends of the scores of each of the six functioning domains of the K-WHODAS II and the sum of them were maintained until about 3 years after the stroke onset, but declined thereafter. The domain of social participation showed an exceptionally earlier decline from 25 months after onset. ANOVA was applied to compare functioning domains among the five groups. 


\section{DISCUSSION}

The necessity of long-term monitoring of functioning status

Comprehensive, intensive, and task-orientated rehabilitation is crucial for recovery in stroke patients. Early stroke recovery occurs within 3 months after onset, and therefore acute rehabilitative treatment reinforces the maximal recovery from stroke impairment in this period. Subacute stroke recovery partially continues after 6 months, and chronic rehabilitative treatment focuses on the maintenance of functional status and on the use compensation techniques. Therefore, the long-term follow-up of functioning for subjects with chronic stroke is important to find out whether patients are able to adjust to their daily lives in a community.

However, there is a lack of reports to assess the longterm status of the functioning of subjects with chronic stroke returning to a community, especially in South Korea.

The recovery of stroke patients is affected by not only the initial severity of brain injury and rehabilitation therapies but also personal background, such as familial support, socio-economic status, and accessibility to rehabilitation facilities [1]. Hence, this current study surveyed the K-WHODAS II to follow-up on the trends of the functioning of stroke patients.

\section{Long-term functioning and contributive factors}

We assessed the extent of recovery in cognitive function and ADL by comparing the K-MMSE and K-MBI at admission and discharge. In this study, the cognitive function and ADL markedly improved through rehabilitative programs. These functional outcomes consequently had long-term effects on the lives of subjects with chronic stroke after discharge, and that was proven by the negative correlations between the K-MMSE and the K-MBI with the total scores of K-WHODAS II in which lower KWHODAS II scores meant a better state of ADL. As stated in previous studies, cognition deficit negatively affected the instrumental ADL and their social life [9], thus resulting in poor long-term functioning. Furthermore, the dependency in daily living and poor functional performances also led to a decline in long-term functioning status [10].

Concerning the personal background, the education level and working status were strongly correlated with functioning in long-term lives after discharge. This suggests that a higher educational level or working status helps patients to sustain better self-care and social life by providing them with more benefits from medical insurance and their higher socio-economic status [1]. Similarly, a previous study reported that stroke patients in a higher socio-economic status received better medical treatment and showed better functional outcomes [2].

A South Korean study had previously reported the positive influence of a family, the most influential social supportive system, on the psychosocial function and ADL of the subjects with stroke [10]. Although the current study showed that marital status had no correlations with total scores of K-WHODAS II, living with a partner still had significantly lower total scores of K-WHODAS II compared with living without a partner thus reinforcing the positive effect of living with a companion on the functioning status of the subjects with chronic stroke.

In addition, gender and age were correlated with the functioning status after discharge. A previous study had reported that elderly patients and female patients were functionally more disable. In fact, the old age was the more impact factor for the functioning status because of its age-related comorbidity that decreased the ADL abilities [11]. Women were shown to be functionally more disabled than men but that was actually because of their old age and comorbidity at stroke onset, rather than gender [11]. The current study showed that the elderly and male patients were correlated with higher total scores of K-WHODAS II. Considering South Korean cultures and its stratified gender roles, we assume that South Korean male patients were unfamiliar with independent households and self-care and were more vulnerable to social isolation because of the cessation of socio-economic activities, which eventually affected their long-term functioning status in a community.

The K-MBI is a practical tool to evaluate ADL, but it is an in-hospital tool to measure the capacity of ADL rather than the actual performance of ADL in a community. Therefore, the K-WHODAS II would be a more informative instrument to evaluate the actual status of the functioning of stroke patients in a community.

The changes of long-term functioning status

Using the K-WHODAS II, we followed up on the changes 
of functioning status for 4 years after the onset of stroke and discovered that functioning status was maintained until 24-36 months but declined thereafter. A previous study published in 2009 reported that the functional independence of stroke patients annually decreased up to 5 years, and 3 years after stroke onset was the point of greatest change [2]. Hence, our study showed similar results to this previous study.

Several factors may cause a decline of the functioning status from 36 months after stroke onset. First, the socioeconomic status of patients and family may be involved in the maintenance of functioning for a certain period of time. A higher socio-economic status motivates patients for rehabilitative treatment and provides them with sufficient rehabilitation services, information about health, and ongoing management of risk factors and chronic conditions [1]. Second, limited accessibility and the supply of community-based rehabilitation may restrict the interventions and thus cause a decline of functioning status.

Our study implies that stroke patients had severe mobility limitation, and that this finding was contradictory to a previous study, which had reported that the patients who received interventions for 6 months after discharge maintained favorable mobility compared with those who received only conventional managements [2]. This might be because of a lacking welfare system in South Korea, including traditional house structures, inconvenient roads, and dependency on the caregivers. Consequently, the renovation of the environmental state, reinforcement of community-based rehabilitation, caregiver education, and regular intervention by physicians after discharge would promote the early detection of changes in the health condition and maintenance of functioning.

Comparison of WHODAS with other disability measures (Why WHODAS?)

Various types of questionnaire have been developed to evaluate the influence of ill health status on one's life and the outcomes of care. Among them, the short form 36 health survey questionnaire (SF-36) and the EuroQoL five dimensions questionnaire (EQ-5D) have occasionally been used for the evaluation of disabilities.

SF-36 is an abbreviated generic measure of 149 questions [12] that evaluates 8 health domains of the general population as a form of patient reported outcome mea- sures (PROM). EQ-5D is an international measure of health that evaluates 5 functioning domains including clinical and economic aspects of the health status. Both are well-known and frequently used but do not evaluate personal background.

Nonetheless, we used WHODAS II in this study because WHODAS II best supports the ICF, the standard of functioning or disability measure. Furthermore, WHODAS can be applied to any kinds of illness, including physical, psychological, or substance-use disorders [3].

Subjects with chronic stroke have difficulties because of not only the disease progression but also concomitant physical, psychological, or substance-use disorders; thus, we applied WHODAS II, which could evaluate overall functioning difficulties in a community.

\section{Future strategy}

The OECD Healthy Care Quality in 2012 reported that South Korea needs to improve its long-term management of cardiovascular diseases, including the prevention, education, and after-discharge rehabilitation [13]. Because of a decline of functioning after 36 months of stroke onset in this study, regular monitoring of functioning or disabilities with a reliable international tool, such as the WHODAS II, would help to improve the effects of long-term management. Furthermore, community-based rehabilitation services with appropriate evaluation and remodeling of environmental structures would ensure maintenance of functioning and the decrease of medical expenditures.

\section{Limitations}

We enrolled stroke patients whose onset was up to 48 months and asked them to complete the K-WHODAS II questionnaire. However, the current study was not a cohort study, hence some limitations were inevitable. First, we could not investigate changes of personal background before and after the stroke onset. Second, we could not clarify the causal relationships between the personal background and the long-term functioning status. We could only find out that some aspects of personal background, particularly having a job and living with a partner, positively affected the functioning status at the time this K-WHODAS II survey was conducted. Thus, it is necessary to carry out a longitudinal study with a long-term follow-up of patients after disease onset. Moreover, it is 
necessary to conduct a future cohort study on the trends of the long-term status of functioning and disability.

Furthermore, we could not rule out the confounding effects of personal background and K-MBI and K-MMSE. The logistic regression should have been applied to evaluate the confounding effects personal background and KMBI and K-MMSE on the total scores of K-WHODAS II.

\section{Conclusion}

The long-term functioning after stroke was related not only with cognitive and motor status in hospital, but also with some salient aspects in the personal background of the patients. K-WHODAS II may be used to monitor functioning status in a community and to assess personal background in subjects with chronic stroke.

\section{CONFLICT OF INTEREST}

No potential conflict of interest relevant to this article was reported.

\section{ACKNOWLEDGMENTS}

This work was supported by a research grant from Inha University.

\section{REFERENCES}

1. Andersen HE, Eriksen K, Brown A, Schultz-Larsen K, Forchhammer BH. Follow-up services for stroke survivors after hospital discharge: a randomized control study. Clin Rehabil 2002;16:593-603.

2. Dhamoon MS, Moon YP, Paik MC, Boden-Albala B, Rundek T, Sacco RL, et al. Long-term functional recovery after first ischemic stroke: the Northern Manhattan Study. Stroke 2009;40:2805-11.

3. Schlote A, Richter M, Wunderlich MT, Poppendick U, Moller C, Schwelm K, et al. WHODAS II with people after stroke and their relatives. Disabil Rehabil 2009;31:855-64.
4. de Pedro-Cuesta J, Alberquilla A, Virues-Ortega J, Carmona M, Alcalde-Cabero E, Bosca G, et al. ICF disability measured by WHO-DAS II in three community diagnostic groups in Madrid, Spain. Gac Sanit 2011;25 Suppl 2:21-8.

5. World Health Organization. Measuring health and disability: manual for WHO Disability Assessment Schedule (WHODAS 2.0). Switzerland: World Health Organization; 2010.

6. Brazier JE, Walters SJ, Nicholl JP, Kohler B. Using the SF-36 and Euroqol on an elderly population. Qual Life Res 1996;5:195-204.

7. Lee HJ, Kim DJ. Cultural adaptation and reliability testing of Korean version of the World Health Organization Disability Assessment Schedule 2.0: 12-item versions. J Korean Soc Phys Med 2011;6:475-88.

8. Andrews G, Kemp A, Sunderland M, Von Korff M, Ustun TB. Normative data for the 12 item WHO Disability Assessment Schedule 2.0. PLoS One 2009;4:e8343.

9. Patel MD, Coshall C, Rudd AG, Wolfe CD. Cognitive impairment after stroke: clinical determinants and its associations with long-term stroke outcomes. J Am Geriatr Soc 2002;50:700-6.

10. Pettersen R, Dahl T, Wyller TB. Prediction of longterm functional outcome after stroke rehabilitation. Clin Rehabil 2002;16:149-59.

11. Kelly-Hayes M, Beiser A, Kase CS, Scaramucci A, D'Agostino RB, Wolf PA. The influence of gender and age on disability following ischemic stroke: the Framingham study. J Stroke Cerebrovasc Dis 2003;12:11926.

12. Organization for Economic Co-operation and Development. OECD health care quality review: Korea. Paris: Organization for Economic Co-operation and Development; 2012.

13. Kang BH. The relationship between family support and activities of daily living abilities for the hemiplegic patients (after stroke). Korean J Rehabil Nurs 2000;3:5-14. 